\title{
Molecular epidemiological investigation to determine the source of a fatal case of serotype $22 \mathrm{~F}$ pneumococcal meningitis
}

\begin{abstract}
Correspondence
Ana Lucia Andrade alssandrade@gmail.com
\end{abstract}

Received 16 November 2011 Accepted 25 January 2012
Juliana Lamaro-Cardoso, ${ }^{1}$ Ana Paula S. de Lemos, ${ }^{2}$ Maria da Glória Carvalho, ${ }^{3}$ Fabiana Cristina Pimenta, ${ }^{3}$ Alexis Roundtree, ${ }^{3}$ Lorena Motta, ${ }^{4}$ Maria Aparecida Vieira, ${ }^{5}$ Sabrina Sgambatti, ${ }^{5}$ Licia Kamila Thörn, ${ }^{4}$ Vicente Pessoa-Junior, ${ }^{4}$ Ruth Minamisava, ${ }^{6}$ Lee H. Harrison, ${ }^{7}$ Bernard W. Beall, ${ }^{3}$ Maria Cristina de Cunto Brandileone ${ }^{2}$ and Ana Lucia Andrade ${ }^{4}$

\footnotetext{
${ }^{1}$ Department of Microbiology, Immunology, Parasitology and Pathology, Federal University of Goiás, Goiânia, Brazil

${ }^{2}$ Adolfo Lutz Institute, São Paulo, Brazil

${ }^{3}$ Respiratory Diseases Branch, Centers for Disease Control and Prevention, Atlanta, GA, USA

${ }^{4}$ Department of Community Health, Federal University of Goiás, Goiânia, Brazil

${ }^{5}$ Pontifical Catholic University of Goiás, Goiânia, Brazil

${ }^{6}$ School of Nursing, Federal University of Goiás, Goiânia, Brazil

${ }^{7}$ Infectious Diseases Epidemiology Research Unit, University of Pittsburgh Graduate School of Public Health and School of 26 Medicine, Pittsburgh, PA, USA
}

\begin{abstract}
A child's death due to pneumococcal meningitis after contracting the disease in an after-school programme prompted an investigation to assess nasopharyngeal (NP) carriage among her contacts. The serotype of the meningitis case isolate was determined, together with the serotypes of the NP specimens of contacts, comprising the case patient's brother, the case patient's afterschool programme contacts and the brother's day-care centre (DCC) contacts. NP swabs from 155 children and 69 adults were obtained. Real-time PCR and conventional multiplex PCR (CMPCR) assays were used to detect pneumococcal carriage and determine serotypes. Brothenriched culture of NP specimens followed by pneumococcal isolation and Quellung-based serotyping were also performed. DNA extracts prepared from cerebrospinal fluid of the index case and from the NP strain isolated from the brother and from one attendee of the brother's DCC were subjected to genotyping. Pneumococcal carriage assessed by real-time PCR and culture was 49.6 and $36.6 \%$, respectively $(P<0.05)$. Twenty-three serotypes were detected using CM-PCR, with serotypes $6 \mathrm{~A} / 6 \mathrm{~B}, 14,19 \mathrm{~F}, 6 \mathrm{C} / 6 \mathrm{D}, 22 \mathrm{~F} / 22 \mathrm{~A}, 23 \mathrm{~F}$ and $11 \mathrm{~A} / 11 \mathrm{D}$ being the most frequent. All eight serotype 22F/22A NP specimens recovered were from children attending the brother's DCC. The meningitis case isolate and the NP carriage isolate from the patient's brother were both serotype $22 \mathrm{~F}$ and shared the same new multilocus sequence type (ST6403) with the attendee of the brother's DCC. CM-PCR proved to be useful for assessing carriage serotype distribution in a setting of high-risk pneumococcal transmission. The causal serotype appeared to be linked to the brother of the case patient and attendees of his DCC.
\end{abstract}

\section{INTRODUCTION}

Streptococcus pneumoniae is the leading cause of invasive bacterial infections in the paediatric population. Children are a presumptive reservoir of $S$. pneumoniae, and the bacteria colonize the nasopharynx, from which the pneumococcus can potentially spread to cause pneumonia and
Abbreviations: $\mathrm{Cl}$, Confidence interval; $\mathrm{CM}-\mathrm{PCR}$, conventional multiplex PCR; CSF, cerebrospinal fluid; DCC, day-care centre; MLST, multilocus sequence typing; NP, nasopharyngeal; OD, odds ratio; ST, sequence type; STGG, skimmed milk/tryptone/glucose/glycerine. 
invasive pneumococcal disease, such as bacteraemia, sepsis and meningitis (Bogaert et al., 2004). Colonization of the nasopharynx begins in the first year of life, and carriage rates peak in the pre-school age group (Hill et al., 2008). Day-care centres (DCCs) typically increase the circulation of pneumococcal strains among children (de Lencastre \& Tomasz, 2002) as crowded conditions facilitate pneumococcal transmission and subsequent disease (Bogaert et al., 2004; Lauderdale et al., 2005).

Previous cases of pneumococcal meningitis in DCCs have been followed by epidemiological investigation of nasopharyngeal (NP) carriage to detect the source of pneumococcal infection using standard methods of culture and serotyping (Cherian et al., 1994; Craig et al., 1999; Lauderdale et al., 2005). Recent PCR-based assays, such as real-time PCR and conventional multiplex PCR (CMPCR), that use a broth-enrichment step improve the detection and serotyping of pneumococci directly from NP specimens (Carvalho et al., 2010).

On 30 June 2008, the Daycare Institution São Francisco de Assis, Goiânia, Brazil, brought to light a case of pneumococcal meningitis in an attendee who was hospitalized in the intensive care unit of a paediatric hospital of the city in a critical clinical condition, by notifying the epidemiology sector of the Department of Transmissible Diseases of the Secretariat of Health of Goiânia, Brazil. At that time, the Secretariat of Health was asked to help with surveillance measures, as the parents of several other attendees were afraid of their children acquiring meningitis. The Secretariat of Health of Goiânia enlisted the epidemiology group of the Federal University of Goiás to conduct a collaborative surveillance investigation to guide appropriate control actions in the DCC. This prompted an investigation that involved assessing NP carriage and serotypes among the contacts of the index case to ascertain the probable source of infection. The index case was a 10year-old female admitted to hospital because of high fever, headache and vomiting for 2 days prior. On examination in the emergency unit, the patient was lethargic and she had a blood pressure of $70 / 20 \mathrm{mmHg}$, temperature of $35.6{ }^{\circ} \mathrm{C}$, pulse of 148 beats $\min ^{-1}$ and a respiratory rate of 36 breaths $\min ^{-1}$. There was no meningeal rigidity and the rest of the neurological examination was normal. Laboratory analysis revealed a haemoglobin level of $12 \mathrm{~g}$ $\mathrm{dl}^{-1}$, serum aspartate aminotransferase level of $125 \mathrm{U} \mathrm{l}^{-1}$, serum creatinine level of $1.7 \mathrm{mg} \mathrm{dl}^{-1}$ and a platelet count of $49000 \mathrm{~mm}^{-3}$. The cerebrospinal fluid (CSF) was clear and showed normal ranges for leukocytes, glucose and proteins, but the culture yielded $S$. pneumoniae serotyped as $22 \mathrm{~F}$ by the Quellung reaction. The isolate was susceptible to all antimicrobials tested (penicillin, ceftriaxone, chloramphenicol, erythromycin, trimethoprim-sulfamethoxazole and vancomycin) using E-test strips (bioMérieux). Upon hospital admission and for the next 3 days, the girl received ceftriaxone $\left(100 \mathrm{mg} \mathrm{kg}^{-1} \mathrm{day}^{-1}\right)$ but, as she did not show clinical improvement, the antimicrobial therapy was switched to cefepime (150 mg kg $\left.{ }^{-1} \mathrm{day}^{-1}\right)$ and vancomycin $\left(30 \mathrm{mg} \mathrm{kg}^{-1} \mathrm{day}^{-1}\right)$. In spite of the supportive measures and antibiotic treatment, the patient's condition worsened and she died on the day 12 of hospital admission.

Real-time PCR and multiplex PCR have been used to assess NP specimens for pneumococcal carriage from previously vaccinated populations (Moreno et al., 2005; Billal et al., 2008; Antonio et al., 2009; Brugger et al., 2009; Carvalho et al., 2010). Here, we employed real-time PCR and CMPCR to identify pneumococci and pneumococcal serotypes from NP swab specimens collected from contacts of the index patient and of her brother in a non-vaccinated population in Brazil, a country where pneumococcal vaccine had not been introduced into the national routine immunization programme at the time of the patient's diagnosis of meningitis. We also examined the genetic relatedness between the pneumococcal meningitis serotype $22 \mathrm{~F}$ index case isolate and the serotype $22 \mathrm{~F}$ pneumococcal carriage strain circulating among the contacts.

\section{METHODS}

The study protocol was approved by the Regional and National Ethical Committee. An investigation of pneumococcal carriage was undertaken in Goiânia, Brazil, soon after the patient died of meningitis, which was caused by $S$. pneumoniae serotype $22 \mathrm{~F}$. We investigated the contacts of the index case and of her brother, which included children attending DCCs and adults who worked at DCCs. Thus, NP swabs were obtained from the patient's brother, the patient's contacts at the after-school institution and the brother's DCC contacts. The majority $(80 \%)$ of the specimens were collected up to a maximum of 60 days after the diagnosis of meningitis.

Collection of NP swabs. NP swabs $(n=224)$, demographic data and clinical history were obtained from 155 children (median age, 54 months; range, 2 months-14 years) attending the after-school care and DCC, and from 69 adult contacts. Collection of the NP swabs started in July 2008, and in the majority of attendees the collection of swabs occurred from 30 days until 60 days after diagnosis of the index case of meningitis.

The specimens were collected with flexible perinasal calcium alginate swabs (Medical Wire), which were placed into vials containing skimmed milk/tryptone/glucose/glycerine (STGG) transport medium (O'Brien et al., 2001) and sent immediately to the Bacteriology Laboratory of the Federal University of Goiás, Brazil. In the laboratory, the wire portion of the swab was cut at the top level of the tube, allowing the calcium alginate portion of the swab to drop into the vial.

Bacterial isolates. The vials containing the NP swabs in STGG were kept frozen at $-70{ }^{\circ} \mathrm{C}$ and sent to the Streptococcus Laboratory, Centers for Disease Control and Prevention, Atlanta, USA, where they were cultivated in a $4-6 \mathrm{~h}$ broth-enrichment step for conventional culture and Quellung testing. DNA was extracted prior to $l y t A$ realtime PCR and conventional PCR serotyping assays, as described previously (Carvalho et al., 2010).

PCR testing. For the real-time PCR and CM-PCR, DNA extracts for $S$. pneumoniae serotyping were obtained as described previously (http://www.cdc.gov/ncidod/biotech/strep/pcr.htm). The pneumococcus-specific real-time PCR targeting the lytA gene was performed according to Carvalho et al. (2007). Sequential CM-PCR comprising a 
Table 1. Characteristics of participant children

\begin{tabular}{|c|c|c|c|}
\hline Variables & $\begin{array}{l}\text { After-school programme of index case } \\
\qquad(n=44)\end{array}$ & $\begin{array}{l}\text { DCC of brother of index case } \\
\qquad(n=111)\end{array}$ & $P$-value \\
\hline \multicolumn{4}{|l|}{ Age (years) } \\
\hline Mean $( \pm S D)$ & $9.8( \pm 1.75)$ & $3.2( \pm 1.11)$ & $<0.001$ \\
\hline Range & $7-13$ & $0-5$ & \\
\hline Males & $23(52.3 \%)$ & $67(60.4 \%)$ & 0.359 \\
\hline $\begin{array}{l}\text { Mean no. of months attending the } \\
\text { corresponding institution/DCC }( \pm \mathrm{SD})\end{array}$ & $27.3( \pm 21.9)$ & $13.6( \pm 10.8)$ & $<0.001$ \\
\hline $\begin{array}{l}\text { No. of children attending other institutions/ } \\
\text { DCCs }\end{array}$ & $24(54.5 \%)$ & $18(16.2 \%)$ & $<0.001$ \\
\hline $\begin{array}{l}\text { Mean no. of months of attendance at other } \\
\text { institution/DCC }( \pm \mathrm{SD})\end{array}$ & $54.3( \pm 22.7)$ & $14.4( \pm 8.3)$ & $<0.001$ \\
\hline Mean no. of household individuals $( \pm \mathrm{SD})$ & $4.6( \pm 3.1)$ & $4.5( \pm 1.7)$ & 0.862 \\
\hline No. households with smokers & $14(31.8 \%)$ & $38(34.2 \%)$ & 0.852 \\
\hline $\begin{array}{l}\text { No. children administered antibiotics during } \\
\text { the previous } 3 \text { months }\end{array}$ & $8(18.2 \%)$ & $59(53.2 \%)$ & $<0.001$ \\
\hline \multicolumn{4}{|l|}{ S. pneumoniae prevalence } \\
\hline Culture & $6(13.6 \%)$ & $65(58.6 \%)$ & $<0.001$ \\
\hline Real-time PCR & $11(25.0 \%)$ & $80(72.1 \%)$ & $<0.001$ \\
\hline
\end{tabular}

total of 40 serotypes was carried out using eight sequential reactions (Pai et al., 2006; Dias et al., 2007; Morais et al., 2007; see http://www. cdc.gov/ncidod/biotech/strep/pcr.htm for latest updates): reaction 1 : 14, 6A/6B, 6C/6D, 23F, 19A, 9V/9A; reaction 2: 19F, 3, 15B/15C, 18 (18A/18B/18C/18F), 7F/7A; reaction 3: 1, 5, 11A/11D, 9N/9L, 17F; reaction 4: $7 \mathrm{C} / 7 \mathrm{~B} / 40,12 \mathrm{~F} / 12 \mathrm{~A} / 12 \mathrm{~B} / 44 / 46,4,38 / 25 \mathrm{~F} / 25 \mathrm{~A}, 23 \mathrm{~A}$; reaction $5: 8,2,34,20,22 \mathrm{~F} / 22 \mathrm{~A}$; reaction $6: 33 \mathrm{~F} / 33 \mathrm{~A} / 37,15 \mathrm{~A} / 15 \mathrm{~F}$, $35 \mathrm{~F} / 47 \mathrm{~F}, 35 \mathrm{~B}, 16 \mathrm{~F}$; reaction $7: 39,23 \mathrm{~B}, 35 \mathrm{~A} / 35 \mathrm{C} / 42$, 13 ; reaction $8: 24$ (24A/24B/24F), 21, 10F/10C/33C, 10A, 31.

Multilocus sequence typing (MLST). The serotype 22F isolates recovered from the index case CSF and the NP specimens of her brother and a 4-year-old DCC contact of the brother were subjected to MLST as described previously (Enright \& Spratt, 1998) with modifications (Moore et al., 2008).

Data analysis. Co-colonization with multiple serotypes was defined by the presence of at least two different capsular types detected in the same NP specimens. Risk factors related to colonization by multiple serotypes were assessed by odds ratio (OR) and the corresponding $95 \%$ confidence interval $(\mathrm{CI}) . P$ values $<0.05$ were considered as statistically significant. All analysis was performed using SPSS version 16.0 (SPSS).

\section{RESULTS}

The proportion of NP colonization in children and adult contacts was 59.4 and $29.0 \%$, respectively $(P<0.05)$. A higher prevalence of pneumococcal carriage was deduced from the broth-enriched real-time PCR method [49.6\% (111/224); $95 \%$ CI 43.0-56.1] compared with brothenrichment culture method [36.6\% (82/224); $95 \%$ CI 30.5-43.1] $(P=0.006)$.

Characteristics and potential risk factors for pneumococcal $\mathrm{NP}$ colonization associated with children from the afterschool programme and DCC are shown in Table 1. Children attending the brother's DCC were generally younger than the case patient's contacts. The median ages of case contacts and the brother's DCC attendees were 10 years $($ variance $=3.14)$ and 3 years $($ variance $=1.23$ ), respectively $(P<0.01)$. The carriage rate observed in the brother's DCC attendees (72.1\%, 95\% CI $63.7-80.9 \%)$ was higher compared with the case's contacts $(25.0 \%, 95 \%$ CI $13.2-40.3 \%)(P<0.01)$.

Twenty-three capsular types were detected using multiplex PCR, with types $6 \mathrm{~A} / 6 \mathrm{~B}, 14,19 \mathrm{~F}, 6 \mathrm{C} / 6 \mathrm{D}, 22 \mathrm{~F} / 22 \mathrm{~A}, 23 \mathrm{~F}$ and $11 \mathrm{~A} / 11 \mathrm{D}$ being the most frequent (Table 2). Although the combinations $6 \mathrm{~A} / 6 \mathrm{~B}, 22 \mathrm{~F} / 22 \mathrm{~A}, 11 \mathrm{~A} / 11 \mathrm{D}$ and $6 \mathrm{C} / 6 \mathrm{D}$ were not resolved by the PCR assay, based on known carriage serotype frequencies obtained in Brazil by conventional culture (Laval et al., 2006; Berezin et al., 2007; Franco et al., 2010 ), the occurrence of $6 \mathrm{D}, 22 \mathrm{~A}$ and $11 \mathrm{D}$ isolates was considered unlikely. In comparison, 20 serotypes were detected using the Quellung reaction on recovered isolates. There was general agreement between the results of the two serotyping methods in the predominant serotypes found; with both methods, serotypes $6 \mathrm{~A} / 6 \mathrm{~B}, 14,19 \mathrm{~F}, 6 \mathrm{C} / 6 \mathrm{D}, 23 \mathrm{~F}$ and $11 \mathrm{~A} / 11 \mathrm{D}$ comprised six of the seven most prevalent serotypes.

Co-colonization by two to four serotypes was detected by PCR in $7.14 \%(16 / 224)$ of the samples, 13 of which were from attendees of both the DCC and the after-school programme. Three NP swab samples with multiple types were found by the Quellung reaction (Fig. 1). Multiplex PCR detected several of the prevalent serotypes in combination with other serotypes. A total of eight serotypes were found in combination with other serotypes (19F, 22F/22A, 14, 6C/6D, 6A/6B, 13, 23F and 11A/11D). Co-colonization was apparent, with the prevalent serotypes $19 \mathrm{~F}$ and $22 \mathrm{~F} / 22 \mathrm{~A}$ being carried in combination with other serotypes in ten and five specimens, respectively. No 
Table 2. Prevalence of carriage of pneumococcal capsular types detected by multiplex PCR and culture isolation/Quellung in 224 contacts of the patient and of her brother

In total, 155 children and 69 adults were tested. NA, Not applicable.

\begin{tabular}{|c|c|c|c|c|c|}
\hline \multirow[t]{2}{*}{ Capsular type(s) } & \multicolumn{2}{|c|}{ Multiplex PCR $^{\star}$} & \multicolumn{2}{|c|}{ Quellung reaction $\dagger$} & \multirow[t]{2}{*}{ Ratio PCR: Quellung } \\
\hline & $n$ & $\%$ & $n$ & $\%$ & \\
\hline 14 & 17 & 7.6 & 10 & 4.7 & 1.7 \\
\hline $19 \mathrm{~F}$ & 14 & 6.3 & 10 & 4.5 & 1.4 \\
\hline $6 \mathrm{C} / 6 \mathrm{D}$ & 13 & 5.8 & $11(6 C)$ & 4.9 & 1.2 \\
\hline $11 \mathrm{~A} / 11 \mathrm{D}$ & 6 & 2.7 & $7(11 \mathrm{~A})$ & 3.1 & 0.9 \\
\hline 13 & 3 & 1.3 & 2 & 0.9 & 1.5 \\
\hline 3 & 2 & 0.9 & 3 & 1.3 & 0.7 \\
\hline $15 B / 15 \mathrm{C}$ & 2 & 0.9 & $1(15 B)$ & 0.4 & 2.0 \\
\hline 4 & 1 & 0.4 & 0 & 0.0 & 1.0 \\
\hline $16 \mathrm{~F}$ & 1 & 0.4 & 0 & 0.0 & 1.0 \\
\hline $18(18 \mathrm{~A} / 18 \mathrm{~B} / 18 \mathrm{C} / 18 \mathrm{~F})$ & 1 & 0.4 & 0 & 0.0 & 1.0 \\
\hline 20 & 1 & 0.4 & 0 & 0.0 & 1.0 \\
\hline $23 \mathrm{~B}$ & 1 & 0.4 & 1 & 0.4 & 1.0 \\
\hline $28 \mathrm{~A}$ & 0 & 0.0 & 1 & 0.4 & 0 \\
\hline $33 \mathrm{~F} / 33 \mathrm{~A} / 37$ & 1 & 0.4 & 0 & 0.0 & 1 \\
\hline $35 \mathrm{~B}$ & 1 & 0.9 & 2 & 0.9 & 0.5 \\
\hline $35 \mathrm{~F} / 47 \mathrm{~F}$ & 1 & 0.4 & $1(35 \mathrm{~F})$ & 0.4 & 1 \\
\hline 39 & 1 & 0.4 & 0 & 0.0 & 1 \\
\hline 34 & 0 & 0.0 & 1 & 0.4 & 0 \\
\hline High $C_{\mathrm{t}}$ for $l y t A \ddagger$ & 17 & 7.6 & NA & NA & NA \\
\hline
\end{tabular}

*Sixteen samples showed co-colonization (two or more serotypes).

†One sample was missing; 11 samples showed co-colonization.

\#Enriched-broth DNA extracts were not PCR typable due to low DNA concentration $\left(\right.$ lytA $\left.C_{\mathrm{t}}>30\right)$.

§Non-typable by multiplex PCR for 40 serotype specificities.

IIEight samples showed co-colonization.

serotype $22 \mathrm{~F}$ was detected in the after-school contacts. However, the meningitis index case and the isolate recovered from the patient's brother were both serotype 22F. Whilst multiplex PCR detected type 22F carriage among eight attendees in the brother's DCC, culture isolation followed by Quellung testing only allowed detection of $22 \mathrm{~F}$ carriage in two individuals.

The same newly described multilocus sequence type, ST6403 (1, 16, 71, 1, 6, 17, 6), was shared between the index case isolate from CSF and the other two NP serotype $22 \mathrm{~F}$ isolates - one from the brother and one from a 4-yearold also attending the brother's DCC. ST6403 is a singlelocus variant of ST698 from two serotype 22F carriage isolates described previously in Australia and the UK (http://www.mlst.net/).
The risk of being colonized by multiple types was higher for young children compared with older children $(\mathrm{OR}=$ $3.0 ; 95 \%$ CI $0.93-9.61 ; P<0.05)$. Children from the brother's DCC (i.e. younger children) were more prone to carry multiple serotypes $(\mathrm{OR}=3.30$; $95 \%$ CI $1.03-$ 10.58) (Table 3).

\section{DISCUSSION}

We traced the possible source of a fatal case of serotype 22F meningitis by using broth-enriched PCR, demonstrating the utility of multiplex PCR in a pneumococcal carriage field investigation.

It is well known that pneumococcal colonization is highest in young children and that pneumococcal disease, especially 


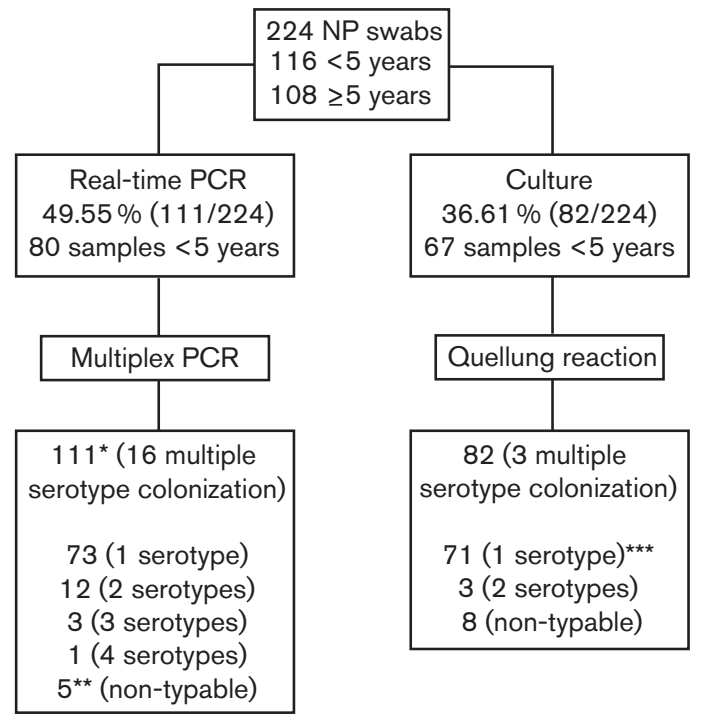

Fig. 1. Detection of pneumococcal carriage and co-colonization by multiplex PCR and Quellung testing. *, Includes 17 samples with a cycle threshold $\left(C_{t}\right)$ of $>30$ for lyt $A$ real-time PCR, which is above the serotyping detection limit by multiplex PCR; ${ }^{\star *}, c p s A$ PCR-positive but non-typable by multiplex PCR for 40 serotype specificities; ${ }^{\star \star *}$, both serotypable and non-typable isolates were obtained in seven of these cultures.

meningitis, is rare in older children. Therefore, cases of pneumococcal illness occurring in older children are likely to be linked to transmission from a younger child. Our investigation and those of others (Brugger et al., 2010) indicate that younger infants may be at greater risk not only of pneumococcal colonization but also colonization by multiple serotypes. Interestingly, no child other than the case patient was colonized by the causal serotype $22 \mathrm{~F}$ in the after-school institution, whilst several 22F-positive specimens were observed among children from the brother's DCC, including a specimen from the case patient's brother. Although these observations strongly suggest that the case patient may have contracted the causal serotype 22F strain from her younger brother, transmission in the other direction cannot be excluded.

The use of culture isolation, instead of multiplex PCR, for assessing pneumococcal carriage serotype distribution appeared to underestimate serotype $22 \mathrm{~F}$ dissemination among the brother's DCC attendees by a factor of four (eight vs two positive specimens; Table 2). However, this approach did allow a comparison of isolates using MLST. It is conceivable that MLST would have been effective in the three culture-negative specimens that yielded only the $22 \mathrm{~F}$ serotype; however, this was not pursued. Although MLST of such enriched-broth specimens would be prevented by co-colonization of multiple pneumococcal strains, it is currently unknown what confounding effects would be contributed by closely related co-colonizing species or by non-typable co-colonizing pneumococci.

Sequential multiplex PCR was used initially for deducing the serotypes of pneumococcal isolates (Pai et al., 2006; Dias et al., 2007; Morais et al., 2007). More recently, multiplex PCR has been utilized for detecting pneumococcal capsular types within NP specimens without recovering isolates (Moreno et al., 2005; Antonio et al., 2009; Carvalho et al., 2010). We found more non-typable pneumococci by Quellung testing than by multiplex PCR. At least two plausible explanations for this can be suggested. The first is that we observed very few colonizing serotypes that are not included within the 40 serotypes that the multiplex PCR is capable of detecting. Basically, there are quite a number of serotypes, more than 92, that are rarely encountered. Secondly, a large fraction of the Quellung non-typable isolates were undoubtedly undetectable in the PCR assay, as many of these lack a cPsA PCR

Table 3. Variables associated with multiple pneumococcal serotypes in 224 carriage isolates of children and adult contacts

\begin{tabular}{|c|c|c|c|c|}
\hline \multirow[t]{2}{*}{ Variables } & \multicolumn{2}{|c|}{ Multiple serotypes by multiplex PCR } & \multirow[t]{2}{*}{ OR $(95 \% \mathrm{CI})$} & \multirow[t]{2}{*}{$P$ value } \\
\hline & Yes $(n=16)$ & No $(n=208)$ & & \\
\hline \multicolumn{5}{|l|}{ Age (years) } \\
\hline$<5$ & 12 & 104 & $3.00(0.93-9.61)$ & 0.048 \\
\hline$\geqslant 5$ & 4 & 104 & & \\
\hline \multicolumn{5}{|l|}{ Sex } \\
\hline Male & 10 & 104 & $1.67(0.58-4.76)$ & 0.332 \\
\hline Female & 6 & 104 & & \\
\hline \multicolumn{5}{|l|}{ Contacts } \\
\hline Brother's DCC & 12 & 99 & $3.30(1.03-10.58)$ & 0.031 \\
\hline Meningitis patient & 4 & 109 & & \\
\hline \multicolumn{5}{|c|}{$\begin{array}{l}\text { Use of antibiotic in the previous } \\
3 \text { months }\end{array}$} \\
\hline Yes & 6 & 82 & $1.08(0.38-3.12)$ & 0.879 \\
\hline No & 10 & 126 & & \\
\hline
\end{tabular}


target, or co-colonize and lack a serotype-specific cps segment, resulting in a masking effect.

The limitations of this study should be also mentioned. It is well known that the duration of colonization can vary with age and serotype. Thus, in this investigation, $20 \%$ of the collected swabs were taken 60 days after the index cases's diagnosis of meningitis. For these cases, it is plausible that the serotype $22 \mathrm{~F}$ clone swept through the attendees in this 'microepidemic' of carriage, or that this 'epidemic' had passed by the time we undertook the investigation, which could have affected the likelihood of detecting serotype 22F. Whilst the patient's brother was swabbed just 15 days after the patient's death, it is interesting to note that, among the other seven detected serotype $22 \mathrm{~F}$ strains, four could still be recovered by NP swabbing $>60$ days after the meningitis case diagnosis. Unfortunately, only one of these children harboured an isolate that we were able to confirm genetic similarity with the meningitis case. Another point to consider is that some attendees from the DCC and afterschool institution may have been exposed to antibiotics in the interval between the start of the investigation and collection of the swab, or were only transiently colonized by serotype $22 \mathrm{~F}$ such that its carriage was not detected. It is also possible that the detected $22 \mathrm{~F}$ carriage was initiated from an outside source after the case had occurred.

A recent publication showed that serotype $22 \mathrm{~F}$ has been found among the most prevalent emerging non-vaccine serotypes in pneumococcal carriage in paediatric outpatients in the UK after 3 years of vaccination with the pneumococcal conjugate vaccine (PCV-7) (Tocheva et al., 2011). In 2010, Brazil became the first country to introduce the 10-valent pneumococcal vaccine into its national immunization programme. The impact of this vaccine on pneumococcal infections and carriage should be assessed using accurate diagnostic tests. Multiplex PCR appears to have great potential use in studies assessing the impact of vaccination on carriage serotype distribution, especially for non-dominant serotypes that may often be 'masked' by cocolonizing pneumococci present in higher densities.

Our findings confirmed that a broth-enrichment step prior to DNA extraction enhances detection of low-density and mixed serotypes, as reported previously (Carvalho et al., 2010). The multiplex PCR approach can be particularly useful in population-based studies where there is limited laboratory capability for taking on the highly demanding work of conventional pneumococcal isolation and serotyping. There is little doubt that standard culture underestimates lowdensity serotypes, especially those present in mixed carriage with other serotypes. It is plausible that a broth-enrichment/ PCR approach will allow more detailed comparisons of serotype distribution changes that occur after vaccination.

\section{ACKNOWLEDGEMENTS}

This study was partially supported by a Fogarty International Center Global Infectious Diseases Research Training Program grant, National
Institutes of Health to the University of Pittsburgh (5D43TW006592) and by the Secretary of Health of Municipality of Goiânia, Goiás, Brazil. A.L.A. and M.C.C.B. are research fellows of The Brazilian National Council for Scientific and Technological 317 Development/ CNPq. (research grant nos 306096/2010-2 and 302175/2010-5, respectively) We thank Robert Gertz and Zhongya Li for MLST results.

\section{REFERENCES}

Antonio, M., Hakeem, I., Sankareh, K., Cheung, Y. B. \& Adegbola, R. A. (2009). Evaluation of sequential multiplex PCR for direct detection of multiple serotypes of Streptococcus pneumoniae from nasopharyngeal secretions. J Med Microbiol 58, 296-302.

Berezin, E. N., Cardenuto, M. D., Ferreira, L. L., Otsuka, M., Guerra, M. L. \& Brandileone, M. C. (2007). Distribution of Streptococcus pneumoniae serotypes in nasopharyngeal carriage and in invasive pneumococcal disease in Sao Paulo, Brazil. Pediatr Infect Dis J 26, 643-645.

Billal, D. S., Hotomi, M., Suzumoto, M., Yamauchi, K., Arai, J., Katsurahara, T., Moriyama, S., Fujihara, K. \& Yamanaka, N. (2008). Determination of pneumococcal serotypes/genotypes in nasopharyngeal secretions of otitis media children by multiplex PCR. Eur J Pediatr 167, 401-407.

Bogaert, D., De Groot, R. \& Hermans, P. W. (2004). Streptococcus pneumoniae colonisation: the key to pneumococcal disease. Lancet Infect Dis 4, 144-154.

Brugger, S. D., Hathaway, L. J. \& Mühlemann, K. (2009). Detection of Streptococcus pneumoniae strain cocolonization in the nasopharynx. J Clin Microbiol 47, 1750-1756.

Brugger, S. D., Frey, P., Aebi, S., Hinds, J. \& Mühlemann, K. (2010). Multiple colonization with $S$. pneumoniae before and after introduction of the seven-valent conjugated pneumococcal polysaccharide vaccine. PLOS ONE 5, e11638.

Carvalho, M. G., Tondella, M. L., McCaustland, K., Weidlich, L., McGee, L., Mayer, L. W., Steigerwalt, A., Whaley, M., Facklam, R. R. \& other authors (2007). Evaluation and improvement of real-time PCR assays targeting lytA, ply, and psaA genes for detection of pneumococcal DNA. J Clin Microbiol 45, 2460-2466.

Carvalho, M. G., Pimenta, F. C., Jackson, D., Roundtree, A., Ahmad, Y., Millar, E. V., O'Brien, K. L., Whitney, C. G., Cohen, A. L. \& Beall, B. W. (2010). Revisiting pneumococcal carriage by use of broth-enrichment and PCR techniques for enhanced detection of carriage and serotypes. J Clin Microbiol 48, 1611-1618.

Cherian, T., Steinhoff, M. C., Harrison, L. H., Rohn, D., McDougal, L. K. \& Dick, J. (1994). A cluster of invasive pneumococcal disease in young children in child care. JAMA 271, 695-697.

Craig, A. S., Erwin, P. C., Schaffner, W., Elliott, J. A., Moore, W. L., Ussery, X. T., Patterson, L., Dake, A. D., Hannah, S. G. \& Butler, J. C. (1999). Carriage of multidrug-resistant Streptococcus pneumoniae and impact of chemoprophylaxis during an outbreak of meningitis at a day care center. Clin Infect Dis 29, 12571264.

de Lencastre, H. \& Tomasz, A. (2002). From ecological reservoir to disease: the nasopharynx, day-care centres and drug-resistant clones of Streptococcus pneumoniae. J Antimicrob Chemother 50 (Suppl. S2), $75-82$.

Dias, C. A., Teixeira, L. M., Carvalho, M. G. \& Beall, B. (2007). Sequential multiplex PCR for determining capsular serotypes of pneumococci recovered from Brazilian children. J Med Microbiol 56, $1185-1188$. 
Enright, M. C. \& Spratt, B. G. (1998). A multilocus sequence typing scheme for Streptococcus pneumoniae: identification of clones associated with serious invasive disease. Microbiology 144, 3049-3060.

Franco, C. M., Andrade, A. L., Andrade, J. G., Almeida e Silva, S., Oliveira, C. R., Pimenta, F. C., Lamaro-Cardoso, J., Brandão, A. P., Almeida, S. C. \& other authors (2010). Survey of nonsusceptible nasopharyngeal Streptococcus pneumoniae isolates in children attending day-care centers in Brazil. Pediatr Infect Dis J 29, 77-79.

Hill, P. C., Cheung, Y. B., Akisanya, A., Sankareh, K., Lahai, G., Greenwood, B. M. \& Adegbola, R. A. (2008). Nasopharyngeal carriage of Streptococcus pneumoniae in Gambian infants: a longitudinal study. Clin Infect Dis 46, 807-814.

Lauderdale, T.-L., Lee, W. Y., Cheng, M. F., Huang, I. F., Lin, Y. C., Hseih, K. S., Huang, I.-W. \& Chiou, C. C. (2005). High carriage rate of high-level penicillin-resistant Streptococcus pneumoniae in a Taiwan kindergarten associated with a case of pneumococcal meningitis. BMC Infect Dis 5, 96.

Laval, C. B., de Andrade, A. L., Pimenta, F. C., de Andrade, J. G., de Oliveira, R. M., Silva, S. A., de Lima, E. C., Fabio, J. L., Casagrande, S. T. \& Brandileone, M. C. (2006). Serotypes of carriage and invasive isolates of Streptococcus pneumoniae in Brazilian children in the era of pneumococcal vaccines. Clin Microbiol Infect 12, 50-55.

Moore, M. R., Gertz, R. E., Jr, Woodbury, R. L., Barkocy-Gallagher, G. A., Schaffner, W., Lexau, C., Gershman, K., Reingold, A., Farley, M. \& other authors (2008). Population snapshot of emergent Streptococcus pneumoniae serotype 19A in the United States, 2005. J Infect Dis 197, 1016-1027.

Morais, L., Carvalho, M. G., Roca, A., Flannery, B., Mandomando, I., Soriano-Gabarró, M., Sigauque, B., Alonso, P. \& Beall, B. (2007). Sequential multiplex PCR for identifying pneumococcal capsular serotypes from South-Saharan African clinical isolates. $J$ Med Microbiol 56, 1181-1184.

Moreno, J., Hernández, E., Sanabria, O. \& Castañeda, E. (2005). Detection and serotyping of Streptococcus pneumoniae from nasopharyngeal samples by PCR-based multiplex assay. J Clin Microbiol 43, 6152-6154.

O’Brien, K. L., Bronsdon, M. A., Dagan, R., Yagupsky, P., Janco, J., Elliott, J., Whitney, C. G., Yang, Y.-H., Robinson, L.-G. \& other authors (2001). Evaluation of a medium (STGG) for transport and optimal recovery of Streptococcus pneumoniae from nasopharyngeal secretions collected during field studies. J Clin Microbiol 39, 10211024.

Pai, R., Gertz, R. E. \& Beall, B. (2006). Sequential multiplex PCR approach for determining capsular serotypes of Streptococcus pneumoniae isolates. J Clin Microbiol 44, 124-131.

Tocheva, A. S., Jefferies, J. M., Rubery, H., Bennett, J., Afimeke, G., Garland, J., Christodoulides, M., Faust, S. N. \& Clarke, S. C. (2011). Declining serotype coverage of new pneumococcal conjugate vaccines relating to the carriage of Streptococcus pneumoniae in young children. Vaccine 29, 4400-4404. 